\title{
Dynamic Time Warping As a Novel Tool in Pattern Recognition of ECG Changes in Heart Rhythm Disturbances
}

\author{
Volkan Tuzcu, MD \\ Arkansas Children`s Hospital, University of \\ Arkansas for Medical Sciences \\ Little Rock, AR, USA \\ tuzcuvolkan@uams.edu
}

\author{
Selman Nas, PhD \\ Arkansas Children`s Hospital, University of \\ Arkansas for Medical Sciences \\ Little Rock, AR, USA \\ nasselman@uams.edu
}

\begin{abstract}
We present a novel method for the classification and identification of electrocardiograms (ECGs) of various heart rhythm disturbances. This is an essential step in the automatic analysis of heart rhythm disturbances. Dynamic time warping (DTW) is used for this purpose. DTW is utilized successfully in speech recognition. Wavelet analysis is used in some implantable cardioverter defibrillators currently for ECG waveform recognition and classification purpose. The simulations of time-series ECG data of various rhythm disturbances are produced. Normal sinus rhythm ECG templates are compared to the simulated rhythms by both methods. DTW analysis successfully differentiates the ECGs of various arrhythmias. Of note, DTW is able to differentiate ventricular tachycardia from supraventricular tachycardia unlike wavelet analysis. Differentiation of these two rhythm types has significant clinical implications. DTW can potentially be used for automatic pattern recognition of ECG changes representative of various rhythm disturbances.
\end{abstract}

Keywords: Pattern recognition, dynamic time warping, wavelet analysis.

\section{Introduction}

Electrocardiogram (ECG) signals from heart beats have typical features such as $\mathrm{P}$ wave, QRS complex, and T wave. QRS complex represent the signal during which the ventricles are depolarized. Heart pump function is realized after the QRS signal stimulates the ventricles. One heart beat can be defined as a period from one $\mathrm{R}$ peak to the next one. ECG signals are quasiperiodic and nonstationary and heart beats have nonlinear characteristics and change over time. QT interval is defined as the time interval between the onset of Q wave and the end of the following $\mathrm{T}$ wave in an ECG signal. Similarly, RR interval is the time interval between the peak of two consecutive $\mathrm{R}$ waves.

Abnormalities of the heart rhythm (arrhythmias), constitute a major problem in patients with various heart diseases. Arrhythmias represent cardiac electrical system abnormalities which can be detected by the ECG changes. One of the leading reasons for death is life threatening ventricular arrhythmias. Ventricular arrhythmias are fast rhythms that originate from the ventricles. One of the most important characteristics of such arrhythmias is that the QRS complexes are wider and also different in shape compared to the QRS complexes that occur during normal sinus rhythm.

Implantable cardiac defibrillators (ICD) can detect and treat such rhythms. Automatic recognition of such rhythms is therefore critically important for ICDs. Currently, most of the ICDs use only rate criteria for detection of life threatening arrhythmias. One of the ICDs use wavelet analysis for QRS complex classification in detection of such significant arrhythmias (Medtronic Inc, Marquis ICDs). However, there is still a need for better ECG discrimination algorithms.

False detection of arrhythmias can result in inappropriate therapy and therefore can lead to significant problems for the patient. Therefore, the ECG detectors have to be extremely robust but also have to meet high performance requirements. Being able to distinguish life threatening arrhythmias from the normal rhythm or more benign arrhythmias with high accuracy is crucial in the cardiac electrophysiology field.

Time-frequency wavelet theory is used for the detection of life threatening arrhythmias. The wavelet analysis is found to be useful in differentiation of ventricular fibrillation, ventricular tachycardia and atrial fibrillation [1]. Dynamic Time Warping (DTW) is a much more robust distance measure for time series, allowing similar shapes to match even if they are out of phase in the time axis. Because of this flexibility, DTW is widely used in science, industry and finance. However, there is an increasing awareness that the Euclidean distance is a very brittle distance measure $[2,3]$. What is needed is a method that allows an elastic shifting of the time axis, to accommodate sequences which are similar, but out of phase, as shown in Figure 3.

DTW, being such a technique, based on dynamic programming, has long been utilized by the speech processing community $[4,5,6]$. 
Dynamic Time Warping is a technique to align two sequences in order to obtain a dissimilarity measure using non-linear temporal alignment. ECG and speech signals have similar nonstationary characteristics [7]. Therefore Euclidean distance is not available to compare the similarity between two ECG frames due to the inconsistency of the frame length. Heart beat variation causes nonlinear time fluctuation of ECG frames. Elimination of this fluctuation by time-normalization is one of the methods for ECG classification research. However, a linear normalization technique in which the timing difference between the ECG frames is minimized, is inherently insufficient for dealing with the highly nonlinear ECG frame fluctuations. On the other hand, the DTW matching is a pattern matching algorithm with a nonlinear time-normalization effect. It has been used successfully in speech recognition for that purpose [8]. In this algorithm, the fluctuation in time is modeled approximately by a nonlinear warping function with some carefully specified properties. Timing difference between two ECG frames is minimized by warping the time axis of one frame such that the maximum coincidence is attained with the other. Then the time-normalization is calculated as the individual minimized residual distance between them. This minimization process is carried out efficiently by the DTW technique [6].

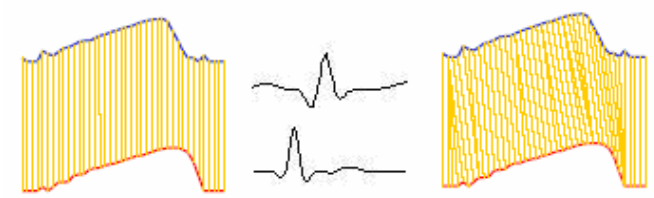

Figure 3. One to one alignment on time axis vs. non-linear alignment (warped time axis). Nonlinear curve alignment is important in pattern recognition of ECG signals. Wavelet analysis dose not allow this type of flexibility in pattern recognition and matching.

\section{Methods}

We use ECGSYN software, which is developed for generation of synthesized ECG [9]. ECGSYN generates a synthesized ECG signal with user-settable mean heart rate, number of beats, sampling frequency, waveform morphology $(\mathrm{P}, \mathrm{Q}, \mathrm{R}, \mathrm{S}$, and $\mathrm{T}$ timing, amplitude, and duration), standard deviation of the RR interval, and LF/HF ratio (a measure of the relative contributions of the low and high frequency components of the RR time series to total heart rate variability). Using a model based on three coupled ordinary differential equations, ECGSYN reproduces many of the features of the human ECG, including beat-to-beat variation in morphology and timing, respiratory sinus arrhythmia, QT dependence on heart rate, and R-peak amplitude modulation. The output of ECGSYN may be employed to assess biomedical signal processing techniques which are used to compute clinical statistics from the ECG.

The algorithms used by ECGSYN are described in [10]. Current implementations of ECGSYN allow the user to modify the morphology of the P-QRS-T cycle, which was not a feature of the original ECGSYN described in the paper. The angle of each attractor $(\mathrm{P}, \mathrm{Q}, \mathrm{R}, \mathrm{S}$ and $\mathrm{T})$ around the limit cycle is set by ti (initially, $\left[\begin{array}{llll}-70 & -15 & 0 & 15\end{array}\right.$ $\left.100]^{*} \pi / 180\right)$. Their positions above or below the $\mathrm{z}=0$ plane are set by bi and the widths of the waveform components are given by ai. Since ti $=0$ defines the placement of the Rpeak, the ordering of each element of ti, ai and bi is [P Q R $\mathrm{S} \mathrm{T}]$. The bi and the ti are stretched by the square root of the reciprocal mean RR interval, as suggested by Bazett's (empirical) formula relating the QT interval to the heart rate. This transformation does not cancel out the reduction of the inter-attractor angular distance that arises 'naturally' from augmented heart rates in this model.

Normal sinus rhythm, two different forms of supraventricular tachycardia, and four different forms of ventricular tachycardia with QRS complexes gradually changing from complexes which resemble normal sinus rhythm complexes to the ones that are very dysmorphic, are generated. All the generated rhythms are carefully analyzed by an cardiac electrophysiologist in order to ensure similarity of the generated ECGs to ECGs that are seen in clinical setting. In supraventricular tachycardia QRS complexes are the same as normal sinus rhythm ones, however $\mathrm{P}$ and $\mathrm{T}$ waves are different and rate is faster. However, in ventricular tachycardia QRS complexes are wider in duration and also different in shape. It is crucial to differentiate between supraventricular tachycardias and ventricular tachycardias for devices like ICDs. Since wavelet analysis is currently used for differentiation purpose in one of the ICD models, besides DTW analysis, we assessed the synthesized time-series ECG data with wavelet analysis as well. ECG time-series data involved 1024 successive beats for each type of rhythm. Normal sinus rhythm ECG template for a single cardiac cycle was compared to each cardiac cycle in these 1024-beat long synthesized rhythms. Each cardiac cycle was extracted from the $\mathrm{R}$ peak to the next $\mathrm{R}$ peak. $\mathrm{R}$ peak represents the peak of the QRS complex. Since the rate is different in each rhythm, we have generated interpolated data points in each beat in order to obtain same number of data points in each cardiac cycle. That way the rate of the rhythm was ignored which also allowed us to interpret only the differences of the ECG pattern of various heart rhythms.

For DTW analysis, we first use a utility to calculate the full local-match matrix i.e. calculating the distance between every pair of frames from the sample and template signals. Then, a simple dynamic programming algorithm that allows three steps - $(1,1),(0,1)$ and $(1,0)$ - with equal weights is used. This approach results in having large constant regions in the modified signals, therefore we 
modified the dynamic programming algorithm to allow $(1,2)$ and $(2,1)$ steps. Let $t$ and $r$ be the template and sample signals and D denotes this local-match matrix at point $(\mathrm{i}, \mathrm{j})$ on a two dimensional grid. The optimum path is found by minimizing this cost function, namely:

$$
D(i, j)=\|t(i)-r(j)\|+\min \left\{\begin{array}{l}
D(i-1, j-2) \\
D(i-1, j-1) \\
D(i-2, j-1)
\end{array}\right\}
$$

The details of the matlab code for the original implementation of DTW can be found in Dan Ellis' Web Page; http://www.ee.columbia.edu/ dpwe/.

The term wavelets refers to sets of function of the form

$$
\psi_{b, a}(t)=|a|^{-1 / 2} \psi\left(\frac{t-b}{a}\right)
$$

i.e., sets of function formed by dilation and translation of a single function, $\psi(t)$ called as the mother wavelet. Continuous wavelet transform on $f(t)$ is defined as

$$
\left(W_{\psi} f(b, a)\right)=\left\langle f(t), \psi_{b, a}(t)\right\rangle=\mid a^{-1 / 2} \int_{-\infty}^{\infty} f(x) \psi\left(\overline{\left.\frac{t-b}{a}\right)} d t\right.
$$

If $(b, a)=\left(\frac{k}{2^{j}}, \frac{1}{2^{j}}\right.$ and $\psi_{j, k}(t)=\psi_{b, a}(t)=2^{j / 2} \psi\left(2^{j} t-k\right)$

then $f(t)$ can be expressed as a wavelet series, that is,

$$
f(t)=\sum_{j, k=-\infty}^{\infty} c_{j, k} \psi_{j, k}(t)
$$

Here,

$$
c_{j, k}=<f(t), \psi_{j, k}(t)>=2^{j / 2} \int_{-\infty}^{\infty} f(t) \overline{\psi\left(2^{j} x-k\right)} d t
$$

The Haar mother wavelet is defined as

$$
\psi(t)=\left\{\begin{array}{l}
1,0 \leq \mathrm{t} \leq 1 \\
-1,0 \leq \mathrm{t} \leq 1 \\
0, \text { otherwise }
\end{array}\right.
$$

The Discrete Wavelet Transform (DWT) is proposed for fast computation. (3) is considered where the discrete values of dilation and translation parameters, $\mathrm{a}$ and $\mathrm{b}$ are used. Dyadic grid is the simplest and most efficient discretization for practical purposes and lends itself to the construction of an orthonormal wavelet basis. Common choices for discrete dyadic grid wavelet parameters $a$ and $b$ is 2 and 1 respectively. The DWT is particularly effective in analyzing waveforms which have spikes or pulses buried in noise. The noise may be more effectively removed and the shape of the pulses preserved. We used Daubechies' first order mother wavelet, which is the same as the Haar mother wavelet, at level six in order to compute the discrete wavelet transformation coefficients. The normalized average distance between the coefficents are used as a measure in order to specify the similarity between two signals.

\section{Results}

A sample of synthetic ECGs representing various types of arrhythmias is shown in Figure 1.

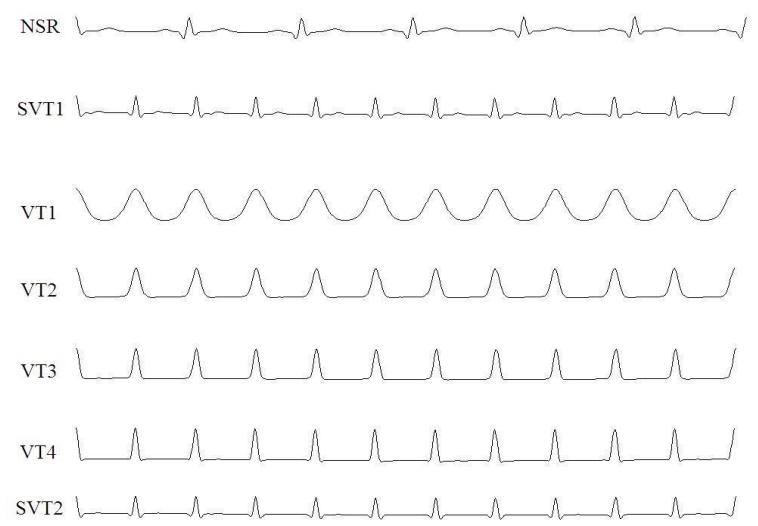

Figure 1. The synthesized ECGs are shown.

In Figure 1, the order of rhythms from top to bottom is as follows: Normal sinus rhythm (NSR), supraventricular tachycardia type 1 (SVT1), ventricular tachycardia type 1 (VT1), ventricular tachycardia type 2 (VT2), ventricular tachycardia type 3 (VT3), ventricular tachycardia type 4 (VT4), and supraventricular tachycardia type 2 (SVT2).

The ventricular tachycardia sample which is labeled as VT4 was closest in morphology to the normal sinus rhythm ECG among the VT forms. However, VT1 represented the most different morphology among the VT forms due to the wider QRS complexes. We also synthesized two different forms of SVT. The ranges of matching of single normal sinus rhythm cycle (one heart beat) template was compared to the 1024 successive beats in each different rhythm and the results are shown in Figure 2.

Data in red color represent results from DTW, and data in green color represent results from wavelet analysis. The order of rhythms is as follows: Normal sinus rhythm, supraventricular tachycardia form 1, ventricular tachycardia form 1, ventricular tachycardia form 2, ventricular tachycardia form 3, ventricular tachycardia form 4, and supraventricular tachycardia form 2. DTW differentiated both types of SVT from all different types of VT successfully without any overlap. However, wavelet analysis demonstrated significant overlap between various forms of VT and SVT. Using wavelet analysis, mean matching percent of NSR to VT4 was $86 \pm 0.49$, and mean 
matching percent of NSR to SVT2 was $87.2 \pm 0.74$. This is interpreted as lack of significant strength in differentiation of SVT from VT since there is significant overlap between two comparisons. However, with DTW analysis, mean matching percent of NSR to VT4 was $36.2 \pm 0.85$ and mean matching percent of NSR to SVT2 was $42.7 \pm 1.34$. DTW allowed discrimination of two rhythms without any significant overlap.

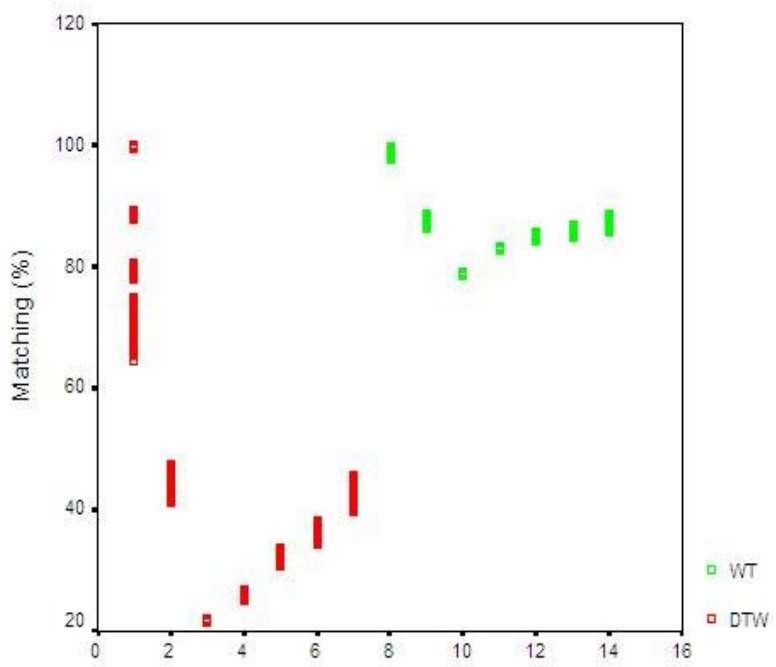

Figure 2. X-axis represents different heart rhythms that were produced. Y-axis represents results of matching ranges of normal sinus rhythm to other rhythms. Single normal sinus rhythm beat template (from $\mathrm{R}$ peak to next $\mathrm{R}$ peak) was compared to 1024 successive beats in each different rhythm. Data in red color represent results from DTW, and data in green color represent results from wavelet analysis. The order of rhythms is as follows: Normal sinus rhythm, supraventricular tachycardia type 1, ventricular tachycardia type 1, ventricular tachycardia type 2, ventricular tachycardia type 3, ventricular tachycardia type 4, and supraventricular tachycardia type 2 .

\section{Discussion}

This study introduces a novel pattern recognition tool for pattern recognition of ECG abnormalities belonging to various heart rhythm disturbances. Although different methods have been used thus far, accurate automatic rhythm recognition is a very important problem in ECG related industry such as in ICDs. Wavelet analysis is the most advanced tool that is being used currently for the automatic recognition of ECG changes in ICDs. However, this feature is not reliable enough and in fact is automatically turned off in ICDs after a certain rate is reached in various rhythm disturbances. Our study proposes that DTW can be a stronger analysis tool for recognition of ECG changes that are noted in lifethreatening arrhythmias. The fact that VT forms that were produced with closer morphology to the normal sinus rhythm template are differentiated with DTW but not with wavelet analysis supports that hypothesis. The most powerful specification of DTW is allowing one to achieve non-linear temporal alignment. Wavelet analysis, however, can only compare time series signals using linear temporal alignment. Due to this lack of flexibility, wavelet analysis could not be used as successfully as DTW in speech recognition. Having similar characteristics to speech signals, ECG signals can also be only be differentiated more succesfully if a nonlinear approach is used in pattern recognition. Therefore, DTW allows one to implement an elastic template matching algorithm by looking for optimal alignment, which seems to be an important strength in assessment of nonstationary time-series signals, such as ECG. DTW is also used in string matching, handwritten character recognition, object recognition, prototype formation, morphing, polygon recognition, curve alignment. Introduction of this technique in ECG analysis has significant potential benefits for clinical electrophysiology.

\section{Conclusions}

DTW is a novel and powerful method in pattern recognition of ECGs that represent various heart rhythm disturbances. DTW can differentiate between normal sinus rhythm, SVT and VT ECGs. However, wavelet analysis seems to have a weaker power in differentiation of ECGs of some arrhythmias. This finding might be very useful in the development of ICDs with better automatic rhythm differentiation algorithms and other ECG related equipment where automatic recognition of various arrhythmias is important.

\section{References}

[1] L. Khadra, A.S. al-Fahoum, and H. al-Nashash, "Detection of life-threatening cardiac arrhythmias using the wavelet transformation," Med Biol Eng Comput., Vol 35, No. 3, pp. 626-632, 1997.

[2] J. Aach and G. Church, "Aligning gene expression time series with time warping algorithms," Bioinformatics, Vol 17, pp. 495-508, 2001.

[3] Z. Bar-Joseph, G. Gerber, D. Gifford, T. Jaakkola, and I. Simon, "A new approach to analyzing gene expression time series data," Proceedings of the 6 Annual International Conference on Research in Computational Molecular Biology, pp. 39-48, 2002.

[4] F. Itakura, "Minimum prediction residual principle applied to speech recognition," IEEE Trans. Acoustics, Speech, and Signal Proc., Vol. ASSP-23, pp. 52-72, 1975. 
[5] J.B. Kruskall and M. Liberman, "The symmetric time warping algorithm: From continuous to discrete. In Time Warps, String Edits and Macromolecules," AddisonWesley, 1983.

[6] H. Sakoe and S. Chiba, "Dynamic programming algorithm optimization for spoken word recognition," IEEE Trans. Acoustics, Speech, and Signal Proc., Vol. ASSP-26, pp. 43-49, 1978.

[7] B. Huang and W. Kinsmer, "ECG frame classification using Dynamic Time Warping," Proc. IEEE Canadian Conference on Electrical \& Computer Engineering, 2002.

[8] H. Sakoe and S. Chiba, "A dynamic programming approach to continuous speech recognition," Proc. Int. Cong. Acous., Budapest, Paper 20C-13, 1971.

[9] A.L. Goldberger, L.A.N. Amaral, L. Glass, J.M. Hausdorff, P.C.H. Ivanov, R.G. Mark, J.E. Mietus, G.B. Moody, C.K. Peng, and H.E. Stanley, "PhysioBank, PhysioToolkit, and PhysioNet: Components of a New Research Resource for Complex Physiologic Signals," Circulation, Vol 101(23), pp. e215-e220, 2000.

[10] P.E. McSharry, G.D. Clifford, L. Tarassenko, L. Smith, "A dynamical model for generating synthetic electrocardiogram signals," IEEE Transactions on Biomedical Engineering, Vol 50, No. 3, pp 289-294, 2003. 